\title{
CHROMATIC NUMBER OF THE PRODUCT OF GRAPHS, GRAPH HOMOMORPHISMS, ANTICHAINS AND COFINAL SUBSETS OF POSETS WITHOUT AC
}

\author{
AMITAYU BANERJEE AND ZALÁN GYENIS
}

\begin{abstract}
In set theory without the Axiom of Choice (AC), we observe new relations of the following statements with weak choice principles.

- If in a partially ordered set, all chains are finite and all antichains are countable, then the set is countable.

- If in a partially ordered set, all chains are finite and all antichains have size $\aleph_{\alpha}$, then the set has size $\aleph_{\alpha}$ for any regular $\aleph_{\alpha}$.

- CS (Every partially ordered set without a maximal element has two disjoint cofinal subsets).

- CWF (Every partially ordered set has a cofinal well-founded subset).

- DT (Dilworth's decomposition theorem for infinite p.o.sets of finite width).

We also study a graph homomorphism problem and a problem due to András Hajnal without AC. Further, we study a few statements restricted to linearly-ordered structures without AC.
\end{abstract}

\section{Introduction}

Firstly, the first author observes the following in ZFA (Zermelo-Fraenkel set theory with atoms).

(1) In Problem 15, Chapter 11 of [KT06, applying Zorn's lemma, Komjáth and Totik proved the statement "Every partially ordered set without a maximal element has two disjoint cofinal subsets" (CS). In Theorem 3.26 of [THS16], Tachtsis, Howard and Saveliev proved that CS does not imply 'there are no amorphous sets' in ZFA. We observe that CS $\nrightarrow A C_{f i n}^{\omega}$ (the axiom of choice for countably infinite familes of non-empty finite sets), CS $\nrightarrow A A C_{n}^{-}$('Every infinite family of $n$-element sets has a partial choice function') 1 for every $2 \leq n<\omega$ and $\mathrm{CS} \not \rightarrow L O K W_{4}^{-}$(Every infinite linearly orderable family $\mathcal{A}$ of 4 -element sets has a partial Kinna-Wegner selection function) 2 in ZFA.

(2) In Problem 14, Chapter 11 of [KT06], applying the well-ordering theorem, Komjáth and Totik proved the statement "Every partially ordered set has a cofinal well-founded subset" (CWF). In Theorem 10(ii) of Tac17, Tachtsis proved that CWF holds in the basic Fraenkel model. Moreover, in Lemma $\mathbf{5}$ of Tac17, Tachtsis proved that CWF is equivalent to $\mathrm{AC}$ in $\mathrm{ZF}$. We observe that $\mathrm{CWF} \not \rightarrow A C_{f i n}^{\omega}, \mathrm{CWF} \not \rightarrow A C_{n}^{-}$for every $2 \leq n<\omega$ and $\mathrm{CWF}$ f $L O K W_{4}^{-}$in ZFA.

(3) In Problem 7, Chapter 11 of [KT06, applying Zorn's lemma, Komjáth and Totik proved that if in a partially ordered set, all chains are finite and all antichains are countable, then the set is countable. We observe that 'If in a partially ordered set, all chains are finite and all antichains are countable, then the set is countable' $\rightarrow A C_{n}^{-}(\forall n \geq$ $2)$, $\rightarrow$ 'There are no amorphous sets' in ZFA which are new results. Moreover, we prove that 'For any regular $\aleph_{\alpha}$, if in a partially ordered set, all chains are finite and all

\footnotetext{
Key words and phrases. Chromatic number of the product of graphs, Graph homomorphisms, Cofinal wellfounded subsets of partially ordered sets, Chains and antichains of partially ordered sets, Linearly-ordered structures, Fraenkel-Mostowski (FM) permutation models of ZFA $+\neg A C$.

${ }^{1}$ It is easy to see that $A C_{n}^{-}$follows from $A C_{n}$ (Axiom of choice for $n$-element sets).

${ }^{2}$ We denote the principle 'Every infinite linearly orderable family $\mathcal{A}$ of $n$-element sets has a partial KinnaWegner selection function' by $L O K W_{n}^{-}$(see HT19]).
} 
antichains have size $\aleph_{\alpha}$, then the set has size $\aleph_{\alpha}$ ' $\nrightarrow \rightarrow A C_{n}^{-}(\forall n \geq 2), \not \rightarrow$ 'There are no amorphous sets' in ZFA.

(4) Dilworth Dil50 proved the following statement: 'If $\mathbb{P}$ is an arbitrary p.o.set, and $k$ is a natural number such that $\mathbb{P}$ has no antichains of size $k+1$ while at least one $k$-element subset of $\mathbb{P}$ is an antichain, then $\mathbb{P}$ can be partitioned into $k$ chains', we abbreviate by DT (see Problem 4, Chapter 11 of [KT06] also). Tachtsis [Tac19] investigated the possible placement of DT in the hierarchy of weak choice principles. He proved that DT does not imply $A C_{f i n}^{\omega}$ as well as $A C_{2}$ (Every family of pairs has a choice function). We observe that DT does not imply $A C_{n}^{-}$for any $2 \leq n<\omega$ in ZFA. In particular, we observe that DT holds in the permutation model of Theorem 8 of [HT19], due to Halbeisen and Tachtsis. We also observe that a weaker form of Łoś's lemma (Form 253 of [HR98]) fails in the permutation model of Theorem 8 of [HT19].

(5) In Theorem 4.5.2 of Kom, Komjáth sketched the following generalization of the $n$ coloring theorem (For every graph $G=(V, E)$ if every finite subgraph of $G$ is $n$-colorable then $G$ is $n$-colorable) applying the Boolean prime ideal theorem (BPI): 'For an infinite graph $G=\left(V_{G}, E_{G}\right)$ and a finite graph $H=\left(V_{H}, E_{H}\right)$, if every finite subgraph of $G$ has a homomorphism into $H$, then so has $G$ ' we abbreviate by $\mathcal{P}_{G, H}$. We observe that if $X \in\left\{A C_{3}, A C_{\text {fin }}^{\omega}\right\}$, then $\mathcal{P}_{G, H}$ restricted to finite graph $H$ with 2 vertices does not imply $X$ in ZFA.

Secondly, we study a weaker formulation of a problem due to András Hajnal in ZFA.

(1) In Theorem 2 of Haj85, Hajnal proved that if the chromatic number of a graph $G_{1}$ is finite (say $k<\omega$ ), and the chromatic number of another graph $G_{2}$ is infinite, then the chromatic number of $G_{1} \times G_{2}$ is $k$ using the Gödel's Compactness theorem. In the solution of Problem 12, Chapter 23 of [KT06], Komjáth provided another argument using the Ultrafilter lemma. For a natural number $k<\omega$, we denote by $\mathcal{P}_{k}$ the following statement.

$$
\text { ' } \chi\left(E_{G_{1}}\right)=k<\omega \text { and } \chi\left(E_{G_{2}}\right) \geq \omega \text { implies } \chi\left(E_{G_{1} \times G_{2}}\right)=k . '
$$

We observe that if $X \in\left\{A C_{3}, A C_{\text {fin }}^{\omega}\right\}$, then $\mathcal{P}_{k} \not \rightarrow X$ in ZFA when $k=3$.

Lastly, we study a few algebraic and graph-theoretic statements restricted to linearly-ordered structures without AC. We abbreviate the statement 'The union of a well-orderable family of finite sets is well-orderable' by $U T(W O, f i n, W O)$. In Theorem 3.1 (i) of [Tac19], Tachtsis proved DT for well-ordered infinite p.o.sets with finite width in ZF applying the following theorem.

Theorem 1.1. (Theorem 1 of [Loeb65]). Let $\left\{X_{i}\right\}_{i \in I}$ be a family of compact spaces which is indexed by a set $I$ on which there is a well-ordering $\leq$. If $I$ is an infinite set and there is a choice function $F$ on the collection $\left\{C: C\right.$ is closed, $C \neq \emptyset, C \subset X_{i}$ for some $\left.i \in I\right\}$, then the product space $\Pi_{i \in I} X_{i}$ is compact in the product topology.

Using the same technique from Theorem 3.1 of [Tac19], we prove a few algebraic and graphtheoretic statements restricted to well-ordered sets, either in $\mathrm{ZF}$ or in $\mathrm{ZF}+U T(W O$, fin, WO $)$. Consequently, those statements restricted to linearly ordered sets are true, in permutation models where LW (Every linearly ordered set can be well-ordered) holds. In particular, we observe the following.

(1) In Theorem 18 of HT13, Howard and Tachtsis obtained that for every finite field $\mathcal{F}=\langle F, \ldots\rangle$, for every nontrivial vector space $V$ over $\mathcal{F}$, there exists a non-zero linear functional $f: V \rightarrow F$ applying BPI. Fix an arbitrary $2 \leq n<\omega$. We observe that 'For every finite field $\mathcal{F}=\langle F, \ldots\rangle$, for every nontrivial linearly-ordered vector space $V$ over $\mathcal{F}$, there exists a non-zero linear functional $f: V \rightarrow F^{\prime} \nrightarrow \rightarrow A C_{f i n}^{\omega}, \not \rightarrow L O K W_{4}^{-}$, and A $A C_{n}^{-}$in ZFA.

(2) Fix an arbitrary $2 \leq n<\omega$. We observe that 'For an infinite graph $G=\left(V_{G}, E_{G}\right)$ on a linearly-ordered set of vertices $V_{G}$ and a finite graph $H=\left(V_{H}, E_{H}\right)$, if every finite 
subgraph of $G$ has a homomorphism into $H$, then so has $G^{\prime} \not \rightarrow A C_{f i n}^{\omega}, \not \rightarrow L O K W_{4}^{-}$, and $\rightarrow A C_{n}^{-}$in ZFA.

(3) Fix an arbitrary $2 \leq n<\omega$. We prove that for every $3 \leq k<\omega$, the statement ' $\mathcal{P}_{k}$ if the graph $G_{1}$ is on some linearly-orderable set of vertices' $t \rightarrow A C_{f i n}^{\omega}, \not \rightarrow L O K W_{4}^{-}$, and $\rightarrow \rightarrow A C_{n}^{-}$in ZFA.

(4) Marshall Hall Hal48, proved that if $S$ is a set and $\left\{S_{i}\right\}_{i \in I}$ is an indexed family of finite subsets of $S$, then if the following property holds,

(P) for every finite $F \subseteq I$, there is an injective choice function for $\left\{S_{i}\right\}_{i \in F}$.

then there is an injective choice function for $\left\{S_{i}\right\}_{i \in I}$. We abbreviate the above assertion by MHT. We recall that BPI implies MHT and MHT implies the Axiom of choice for finite sets $\left(A C_{\text {fin }}\right)$ in ZF (c.f. HR98). Fix an arbitrary $2 \leq n<\omega$. We prove that MHT restricted to a linearly-ordered collection of finite subsets of a set does not imply $A C_{n}^{-}$in ZFA.

\section{A List OF FORMS AND DEFInitions}

(1) The Axiom of Choice, AC (Form 1 in [HR98]): Every family of nonempty sets has a choice function.

(2) The Axiom of Choice for Finite Sets, $A C_{n}$ (Form 62 in [HR98]): Every family of non-empty nite sets has a choice function.

(3) $A C_{2}$ (Form 88 in HR98): Every family of pairs has a choice function.

(4) $A C_{n}$ for each $n \in \omega, n \geq 2$ (Form 61 in HR98): Every family of $n$ element sets has a choice function. We denote by $A C_{n}^{-}$the statement 'Every infinite family of n-element sets has a partial choice function' (Form 342(n) in HR98, denoted by $C_{n}^{-}$in Definition 1 (2) of [HT19]). We denote by $L O K W_{n}^{-}$the statement 'Every infinite linearly orderable family $\mathcal{A}$ of n-element sets has a partial Kinna-Wegner selection function' (c.f. Definition 1 (2) of [HT19).

(5) $A C_{n}^{\omega}$ (Form 10 in [HR98]): Every countably innite family of non-empty nite sets has a choice function. We denote by $P A C_{f i n}^{\omega}$ the statement 'Every countably innite family of non-empty nite sets has a partial choice function'.

(6) The Principle of Dependent Choice, DC (Form 43 in [HR98): If $S$ is a relation on a non-empty set $A$ and $(\forall x \in A)(\exists y \in A)(x S y)$ then there is a sequence $a_{0}, a_{1}, \ldots$ of elements of $A$ such that $(\forall n \in \omega)(a(n) S a(n+1))$.

(7) LW (Form 90 in [HR98): Every linearly-ordered set can be well-ordered.

(8) UT(WO, WO, WO) (Form 231 in [HR98]): The union of a well-ordered collection of well-orderable sets is well-orderable.

(9) UT(WO, fin, WO) (Form 10N in HR98): The union of a well-orderable family of finite sets is well-orderable.

(10) $(\forall \alpha) U T\left(\aleph_{\alpha}, \aleph_{\alpha}, \aleph_{\alpha}\right)$ (Form 23 in [HR98]): For every ordinal $\alpha$, if $A$ and every member of $A$ has cardinality $\aleph_{\alpha}$, then $|\cup A|=\aleph_{\alpha}$.

(11) $\mathbf{U T}\left(\aleph_{0}\right.$, fin, $\left.\aleph_{0}\right)$ (Form 10A in HR98): The union of a denumerable collection of finite sets is countable.

(12) The Boolean Prime Ideal Theorem, BPI (Form 14 in HR98): Every Boolean algebra has a prime ideal. We recall the following equivalent formulations of BPI.

- (Form 14AW in HR98]): The Compactness theorem for propositional logic.

- The Ultrafilter lemma, UL (Form 14A in [HR98]): Every proper filter over a set $S$ in $\mathcal{P}(S)$ can be extended to an ultrafilter.

- The $\boldsymbol{n}$-coloring theorem for $n \geq 3$, (Form 14G $(n)(n \in \omega, n \geq 3)$ in [HR98): For every graph $G=(V, E)$ if every finite subgraph of $G$ is $n$-colorable then $\mathrm{G}$ is $n$-colorable. This is De Bruijn-Erdös theorem for $n \geq 3$ colorings.

(13) The Principle of consistent choice, PCC (Form 14AH in [HR98): Let $\mathcal{A}=$ $\left\{A_{i}\right\}_{i \in I}$ be a family of finite sets and $\mathcal{R}$ is a symmetric binary relation on $\cup_{i \in I} A_{i}$. Suppose that for every finite $W \subset I$, there is an $\mathcal{R}$-consistent choice function for $\left\{A_{i}\right\}_{i \in W}$, then there is an $\mathcal{R}$-consistent choice function for $\left\{A_{i}\right\}_{i \in I}$. 


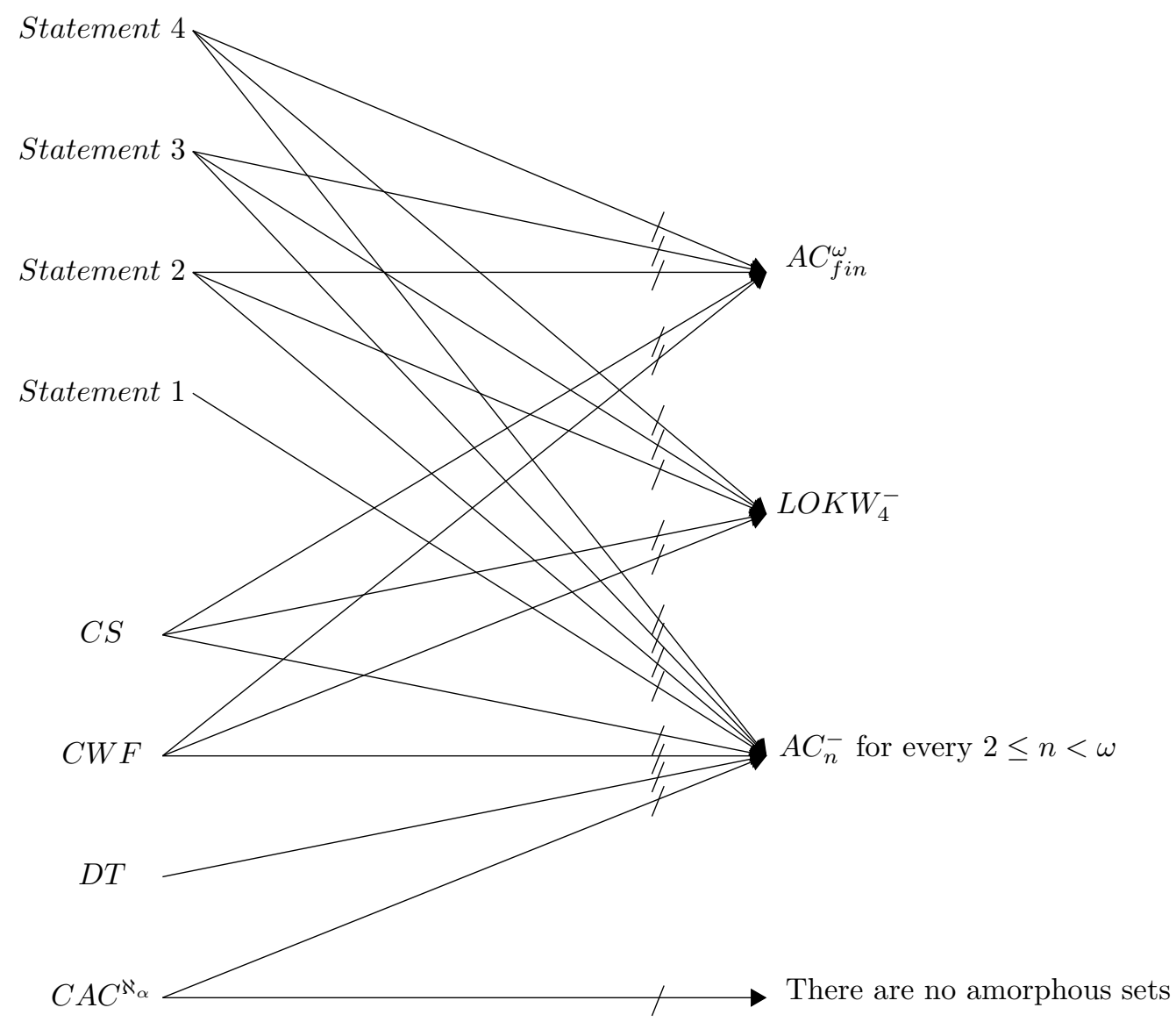

$\mathcal{P}_{G, H}$ restricted to finite graph $H$ with 2 vertices, $\mathcal{P}_{3} \nrightarrow A C_{3}, A C_{\text {fin }}^{\omega}$

FIGURE 1. In the above figure, we sketch the results of this note in ZFA. For each regular $\aleph_{\alpha}$, we denote by $C A C^{\aleph_{\alpha}}$ the statement 'if in a partially ordered set, all chains are finite and all antichains have size $\aleph_{\alpha}$, then the set has size $\aleph_{\alpha}$ '. We use Statement 4 to denote ' $\mathcal{P}_{k}$ for the graph $G_{1}$ on some linearlyorderable set of vertices' for a natural number $k \geq 3$. We use Statement 3 to denote 'For every finite field $\mathcal{F}=\langle F, \ldots\rangle$, for every nontrivial linearly-ordered vector space $V$ over $\mathcal{F}$, there exists a non-zero linear functional $f: V \rightarrow F$ '. We use Statement 2 to denote 'For an infinite graph $G=\left(V_{G}, E_{G}\right)$ on a linearly-ordered set of vertices $V_{G}$ and a finite graph $H=\left(V_{H}, E_{H}\right)$, if every finite subgraph of $G$ has a homomorphism into $H$, then so has $G$ '. We use Statement 1 to denote Marshall Hall's theorem for linearly-ordered collection of finite subsets of a set.

We note that Form 14AH in HR98 is different than the above formulation. Łoś/RyllNardzewski LN51 introduced both the formulations where it was noted that they are equivalent. Let $n \in \omega \backslash\{0,1\}$. We recall the notation $F_{n}$ introduced by Cowen in Cow77, which is PCC restricted to families $\mathcal{A}=\left\{A_{i}: i \in I\right\}$, where $\left|A_{i}\right| \leq n$ for all $i \in I$.

(14) Marshall Hall's theorem, MHT (Form 107 in HR98): If $S$ is a set and $\left\{S_{i}\right\}_{i \in I}$ is an indexed family of finite subsets of $S$, then if the following property holds,

(P) for every finite $F \subseteq I$, there is an injective choice function for $\left\{S_{i}\right\}_{i \in F}$. then there is an injective choice function for $\left\{S_{i}\right\}_{i \in I}$. 
Philip Hall's theorem states that the property $(\mathrm{P})$ is equivalent to the Hall's condition which states that ' $\forall F \in[I]^{<\omega},\left|\cup_{i \in F} S_{i}\right| \geq|F|$ '. We recall that Philip Hall's theorem or finite Hall's theorem can be proved in ZF without using any choice principles.

(15) A weaker form of Loś's lemma, LT (Form 253 in HR98): If $\mathcal{A}=\left\langle A, \mathcal{R}^{\mathcal{A}}\right\rangle$ is a non-trivial relational $\mathcal{L}$-structure over some language $\mathcal{L}$, and $\mathcal{U}$ be an ultrafilter on a non-empty set $I$, then the ultrapower $\mathcal{A}^{I} / \mathcal{U}$ and $\mathcal{A}$ are elementarily equivalent.

(16) MCC (c.f. Definition 5 and Definition 6 of [Tac17]): Every topological space with the minimal cover property is compact.

(17) Bounded and unbounded amorphous sets: An innite set $X$ is called amorphous if $X$ cannot be written as a disjoint union of two innite subsets. There are two types of amorphous sets, namely bounded amorphous sets and unbounded amorphous sets. Let $\mathcal{U}$ be a finitary partition of an amorphous set $X$. Then all but finitely many elements of $\mathcal{U}$ have the same cardinality, say $n(\mathcal{U})$. Let $\Pi(X)$ be the set of all finitary partitions of $X$ and $n(X)=\sup \{n(\mathcal{U}): \mathcal{U} \in \Pi(X)\}$. If $n(X)$ is finite, then $X$ is called bounded amorphous and if $n(X)$ is infinite, then $X$ is called unbounded amorphous. We recall Theorem 6 of [Tac17] which states that MCC $\rightarrow$ "there are no bounded amorphous sets".

(18) (Form 64 in HR98): There are no amorphous sets.

(19) Martin's Axiom (c.f. Tac16b]): If $\kappa$ is a well-ordered cardinal, we denote by $M A(\kappa)$ the principle 'If $(P,<)$ is a nonempty, c.c.c. quasi order and $\mathcal{D}$ is a family of $\leq \kappa$ dense sets in $P$, then there is a filter $\mathcal{F}$ of $P$ such that $\mathcal{F} \cap D \neq \emptyset$ for all $D \in \mathcal{D}^{\prime}$. We recall from Remark 2.7 of [Tac16b] that $A C_{\text {fin }}^{\omega}+M A\left(\aleph_{0}\right) \rightarrow$ 'for every infinite set $\mathrm{X}, 2^{X}$ is Baire' and 'for every infinite set X, $2^{X}$ is Baire' $\rightarrow$ 'there are no amorphous sets'.

(20) Dilworth's decomposition theorem for infinite p.o.sets of finite width, DT (c.f. Tac19): If $\mathbb{P}$ is an arbitrary p.o.set, and $k$ is a natural number such that $\mathbb{P}$ has no antichains of size $k+1$ while at least one $k$-element subset of $\mathbb{P}$ is an antichain, then $\mathbb{P}$ can be partitioned into $k$ chains. We abbreviate the above formulation as DT. We recall Theorem 3.1(i) of [Tac19], which states that DT for well-ordered infinite p.o.sets with finite width is provable in $\mathrm{ZF}$.

(21) The Chain/Antichain Principle, CAC (Form 217 in HR98): Every infinite p.o.set has an infinite chain or an infinite antichain. We recall that $C A C$ implies $A C_{\text {fin }}^{\omega}$ from Lemma 4.4 of Tac19a].

(22) CS (c.f. [THS16]): Every partially ordered set without a maximal element has two disjoint cofinal subsets.

(23) CWF (c.f. Definition 6 (11) of [Tac17]): Every partially ordered set has a cofinal well-founded subset.

(24) Chromatic number of the product of graphs: We recall a few basic terminologies of graphs. An independent set is a set of vertices in a graph, no two of which are connected by an edge. A good coloring of a graph $G=\left(V_{G}, E_{G}\right)$ with a color set $C$ is a mapping $f: V_{G} \rightarrow C$ such that for every $\{x, y\} \in E_{G}, f(x) \neq f(y)$. The chromatic number $\chi\left(E_{G}\right)$ of a graph $G=\left(V_{G}, E_{G}\right)$ is the smallest cardinal $\kappa$ such that the graph $G$ can be colored by $\kappa$ colors. We define the cartesian product of two graphs $G_{1}=\left(V_{G_{1}}, E_{G_{1}}\right)$ and $G_{2}=$ $\left(V_{G_{2}}, E_{G_{2}}\right)$ as the graph $G_{1} \times G_{2}=\left(V_{G_{1} \times G_{2}}, E_{G_{1} \times G_{2}}\right)=\left(V_{G_{1}} \times V_{G_{2}},\left\{\left\{\left(x_{0}, x_{1}\right),\left(y_{0}, y_{1}\right)\right\}\right.\right.$ : $\left.\left.\left\{x_{0}, y_{0}\right\} \in E_{G_{1}},\left\{x_{1}, y_{1}\right\} \in E_{G_{2}}\right\}\right)$ where $V_{G_{1}} \times V_{G_{2}}$ is the cartesian product of the vertex sets $V_{G_{1}}$ and $V_{G_{2}}$. It can be seen that $\chi\left(E_{G_{1} \times G_{2}}\right) \leq \min \left(\chi\left(E_{G_{1}}\right), \chi\left(E_{G_{2}}\right)\right)$. In particular, if $\chi\left(E_{G_{1}}\right)=k<\omega$ then $\chi\left(E_{G_{1} \times G_{2}}\right)=k$, since if $f: V_{G_{1}} \rightarrow\{1, \ldots, k\}$ is a good $k$-coloring of $G_{1}$, then $F(\langle x, y\rangle)=f(x)$ is a good $k$-coloring of $G_{1} \times G_{2}$. In Theorem 2 of Haj85, Hajnal proved that if $\chi\left(E_{G_{1}}\right)$ is finite (say $\left.k<\omega\right)$, and $\chi\left(E_{G_{2}}\right)$ is infinite, then $\chi\left(E_{G_{1} \times G_{2}}\right)$ is $k$. For a natural number $k<\omega$, we denote by $\mathcal{P}_{k}$ the following statement.

$$
\text { ' } \chi\left(E_{G_{1}}\right)=k<\omega \text { and } \chi\left(E_{G_{2}}\right) \geq \omega \text { implies } \chi\left(E_{G_{1} \times G_{2}}\right)=k \text {.' }
$$

2.1. Permutation models. Let $M$ be a model of $Z F A+A C$ where $A$ is a set of atoms or ur-elements. Each permutation $\pi: A \rightarrow A$ extends uniquely to a permutation of $\pi^{\prime}: M \rightarrow M$ by $\epsilon$-induction. Let $\mathcal{G}$ be a group of permutations of $A$ and $\mathcal{F}$ be a normal filter of subgroups of $\mathcal{G}$. 
For $x \in M$, we denote the symmetric group with respect to $\mathcal{G}$ by $\operatorname{sym}_{\mathcal{G}}(x)=\{g \in \mathcal{G} \mid g(x)=x\}$. We say $x$ is $\mathcal{F}$-symmetric if sym $_{\mathcal{G}}(x) \in \mathcal{F}$ and $x$ is hereditarily $\mathcal{F}$-symmetric if $x$ is $\mathcal{F}$-symmetric and each element of transitive closure of $x$ is symmetric. We define the permutation model $\mathcal{N}$ with respect to $\mathcal{G}$ and $\mathcal{F}$, to be the class of all hereditarily $\mathcal{F}$-symmetric sets. It is well-known that $\mathcal{N}$ is a model of $Z F A$ (see Theorem 4.1 of Jec73]). If $\mathcal{I} \subseteq \mathcal{P}(A)$ is a normal ideal, then the set $\left\{\right.$ fix $\left._{\mathcal{G}} E: E \in \mathcal{I}\right\}$ generates a normal filter over $\mathcal{G}$. Let $\mathcal{I}$ be a normal ideal generating a normal filter $\mathcal{F}_{\mathcal{I}}$ over $\mathcal{G}$. Let $\mathcal{N}$ be the permutation model determined by $\mathcal{M}, \mathcal{G}$, and $\mathcal{F}_{\mathcal{I}}$. We say $E \in \mathcal{I}$ supports a set $\sigma \in \mathcal{N}$ if $\operatorname{fix}_{\mathcal{G}} E \subseteq \operatorname{sym}_{\mathcal{G}}(\sigma)$.

\section{WELL-ORDERED STRUCTURES IN ZF}

3.1. Applications of Loeb's theorem. We recall the following fact from Ker00.

Lemma 3.1. $(Z F)$. If $X$ is well-orderable, then $2^{X}$ is compact.

Remark. We can also prove Lemma 3.1 applying Theorem 1 of Loeb65.

Observation 3.2. UT $(W O$, fin, WO) implies Marshall Hall's theorem for any well-ordered collection of finite subsets of a set.

Proof. Let $S$ be a set and $\left\{S_{i}\right\}_{i \in I}$ be a well-ordered indexed family of finite subsets of $S$ such that the following property holds,

(P) for every finite $F \subseteq I$, there is an injective choice function for $\left\{S_{i}\right\}_{i \in F}$.

We work with the propositional language $\mathcal{L}$ with the following sentence symbols.

$$
A_{i, j}^{\prime} \text { where } j \in S_{i} \text { and } i \in I \text {. }
$$

Let $\mathcal{F}$ be the set of all formulae of $\mathcal{L}$ and $\Sigma \subset \mathcal{F}$ be the collection of the following formulae.

(1) $\neg\left(A_{i, m}^{\prime} \wedge A_{j, m}^{\prime}\right)$ for $i \neq j, m \in S_{i} \cap S_{j}$.

(2) $\neg\left(A_{i, j}^{\prime} \wedge A_{i, l}^{\prime}\right)$ for any $l \neq j \in S_{i}$ where $i \in I$.

(3) $A_{i, y_{1}}^{\prime} \vee A_{i, y_{2}}^{\prime} \ldots \vee A_{i, y_{k}}^{\prime}$ for each $i \in I$ where $S_{i}=\left\{y_{1}, \ldots y_{k}\right\}$.

We enumerate $\operatorname{Var}=\left\{A_{i, j}^{\prime}: i \in I, j \in S_{i}\right\}$ since each $S_{i}$ is finite, $I$ is well-orderable and $U T(W O$, fin,$W O)$ is assumed. For every $W \in[I]^{<\omega} \backslash\{\emptyset\}$, we let $\Sigma_{W}$ be the subset of $\mathcal{F}$, which is defined as $\Sigma$ except that the subscripts in the formulae are from the set $W \cup \bigcup_{i \in W} S_{i}$. Endow the discrete 2-element space $\{0,1\}$ with the discrete topology and consider the product space $2^{V a r}$ with the product topology. Let $F_{W}=\left\{f \in 2^{V a r}: \forall \phi \in \Sigma_{W}\left(f^{\prime}(\phi)=1\right)\right\}$ where for $f \in 2^{V a r}$, the element $f^{\prime}$ of $2^{\mathcal{F}}$ denotes the valuation mapping determined by $f$. By Philip Hall's theorem which is provable in ZF without using any choice principles, each $F_{W}$ is nonempty and the family $\mathcal{X}=\left\{F_{W}: W \in[I]^{<\omega} \backslash\{\emptyset\}\right\}$ has the finite intersection property. Also for each $W \in[I]^{<\omega} \backslash\{\emptyset\}, F_{W}$ is closed in the topological space $2^{V a r}$. By Lemma 3.1 since $2^{\text {Var }}$ is compact in ZF, $\cap \mathcal{X}$ is non-empty. Pick an $f \in \cap \mathcal{X}$ and let $f^{\prime} \in 2^{\mathcal{F}}$ be the unique valuation mapping that extends $f$. Clearly, $f^{\prime}(\phi)=1$ for all $\phi \in \Sigma$. Consequently, we obtain an injective choice function for $\left\{S_{i}\right\}_{i \in I}$ by the following claim.

claim 3.3. If $v$ is a truth assignment which satisfies $\Sigma$, then we can define a system of distinct representatives by

$$
y \in S_{i} \text { if and only if } v\left(A_{i, y}^{\prime}\right)=T .
$$

Proof. By (2) and (3) for each $i \in I$, each collection $S_{i}$ gets assigned a unique representative. By (1), distinct sets $S_{i}$ and $S_{j}$ gets assigned distinct representatives. 
Banaschewski Bana92 proved the uniqueness of the algebraic closure of an arbitrary field applying BPI 3

Observation 3.4. $U T(W O$, fin, $W O)$ implies 'If a field $\mathcal{K}$ has an algebraic closure, and the ring of polynomials $\mathcal{K}[x]$ is well-orderable, then the algebraic closure is unique'.

Proof. Let $\mathcal{K}$ be a field, and suppose $\mathcal{E}$ and $\mathcal{F}$ be two algebraic closures of $\mathcal{K}$. We prove that there is an isomorphisn from $\mathcal{E}$ onto $\mathcal{F}$ which fix $\mathcal{K}$ pointwise. Let $\mathcal{E}_{u}$ and $\mathcal{F}_{u}$ be the splitting fields of $u \in \mathcal{K}[x]$ inside $\mathcal{E}$ and $\mathcal{F}$ respectively. Let $\mathcal{H}_{u}$ be the set of all isomorphisms from $\mathcal{E}_{u}$ onto $\mathcal{F}_{u}$ which fix $\mathcal{K}$. Clearly, $\mathcal{H}_{u}$ is a non-empty, finite set. Also, we can see that $\cup_{u} \mathcal{E}_{u}=\mathcal{E}$ and $\cup_{u} \mathcal{F}_{u}=\mathcal{F}$. Let $\mathcal{H}=\Pi_{u \in \mathcal{K}[x]} \mathcal{H}_{u}$, and if $v \mid w$ define $H_{v, w}=\left\{\left(h_{u}\right) \in \mathcal{H}: h_{v}=h_{w}\left\lceil E_{v}\right\}\right.$. Clearly, $H_{v, w}$ has finite intersection property and they are closed in the product topology of $\mathcal{H}$, where each $\mathcal{H}_{u}$ is discrete. Since $\mathcal{K}[x]$ is well-orderable as assumed and for each $u \in \mathcal{K}[x]$, $\mathcal{H}_{u}$ is finite, we have that $\cup_{u \in \mathcal{K}[x]} \mathcal{H}_{u}$ is well-orderable by $U T(W O$, fin, $W O)$. By Theorem 1 of [Loeb65], $\mathcal{H}$ is compact. Consequently, $\bigcap_{v \mid w} \mathcal{H}_{v, w} \neq \emptyset$ and each $\left(h_{u}\right)$ in this intersection determines a unique embedding $h: \cup_{u} \mathcal{E}_{u} \rightarrow \cup_{u} \mathcal{F}_{u}$ which is onto and fixes $\mathcal{K}$.

Observation 3.5. The statement 'For an infinite graph $G=\left(V_{G}, E_{G}\right)$ on a well-ordered set of vertices $V_{G}$ and a finite graph $H=\left(V_{H}, E_{H}\right)$, if every finite subgraph of $G$ has a homomorphism into $H$, then so has $G^{\prime}$ is provable in $\mathrm{ZF}$.

Proof. Fix a finite graph $H=\left(V_{H}, E_{H}\right)$ and a graph $G=\left(V_{G}, E_{G}\right)$ on a well-ordered set of vertices $V_{G}$. We consider $V_{H}=\left\{v_{1}, \ldots v_{k}\right\}$ for some $k<\omega$. We work with the propositional language $\mathcal{L}$ with the following sentence symbols.

$$
A_{x_{i}, v_{j}}^{\prime} \text { where } v_{j} \in V_{H} \text { and } x_{i} \in V_{G} \text {. }
$$

Let $\mathcal{F}$ be the set of all formulae of $\mathcal{L}$ and $\Sigma \subset \mathcal{F}$ be the collection of the following formulae.

(1) $A_{x_{i}, v_{m}}^{\prime} \wedge A_{x_{j}, v_{l}}^{\prime}$ if and only if $\left\{x_{i}, x_{j}\right\} \in E_{G}$ implies $\left\{v_{m}, v_{l}\right\} \in E_{H}$.

(2) $\neg\left(A_{x_{i}, v_{j}}^{\prime} \wedge A_{x_{i}, v_{l}}^{\prime}\right)$ for any $v_{l}, v_{j} \in V_{H}$ such that $v_{l} \neq v_{j}$ and each $x_{i} \in V_{G}$.

(3) $A_{x_{i}, v_{1}}^{\prime} \vee A_{x_{i}, v_{2}}^{\prime} \ldots \vee A_{x_{i}, v_{k}}^{\prime}$ for each $x_{i} \in V_{G}$.

By our assumption $V_{G}$ is well-orderable and $V_{H}$ is finite. So $V_{H}$ is well-orderable. Consequently, $V_{G} \times V_{H}$ is well-orderable in ZF. We enumerate $\operatorname{Var}=\left\{A_{x_{i}, v_{j}}^{\prime}: x_{i} \in V_{G}, v_{j} \in V_{H}\right\}$. By assumption, for every $s \in\left[V_{G}\right]^{<\omega}$ there is a homomorphism $f_{s}: G \nmid s \rightarrow H$ of $G \uparrow s$ into $H$. Following the methods used in the proof of Observation 3.2, we may obtain a $f^{\prime} \in 2^{\mathcal{F}}$ such that $f^{\prime}(\phi)=1$ for all $\phi \in \Sigma$. Consequently, we can obtain a homomorphism $h$ from $G$ to $H$.

Observation 3.6. The statement ' For every finite field $\mathcal{F}=\langle F, \ldots\rangle$, for every nontrivial wellordered vector space $V$ over $\mathcal{F}$, there exists a non-zero linear functional $f: V \rightarrow F$ ' is provable in $\mathrm{ZF}$.

Proof. We follow the proof of Theorem 18 of HT13 and modify it in the context of wellorderable vector space. Fix a finite field $\mathcal{F}=\langle F, \ldots\rangle$ where $F=\left\{v_{1}, \ldots v_{k}\right\}$ and a nontrivial well-ordered vector space $V$ over $\mathcal{F}$. We work with the propositional language $\mathcal{L}$ with the following sentence symbols.

$$
A_{x_{i}, v_{j}}^{\prime} \text { where } v_{j} \in F \text { and } x_{i} \in V \text {. }
$$

Let $\mathcal{F}^{\prime}$ be the set of all formulae of $\mathcal{L}$ and $\Sigma \subset \mathcal{F}^{\prime}$ be the collection of the following formulae.

(1) $A_{a, 1}^{\prime}$

(2) $A_{x_{i}, v_{j}}^{\prime} \rightarrow A_{v_{k} x_{i}, v_{k} v_{j}}^{\prime}$ for $v_{k}, v_{j} \in F$ and $x_{i} \in V$.

(3) $A_{x_{i}, v_{j}}^{\prime} \wedge A_{x_{i^{\prime}}, v_{j^{\prime}}}^{\prime} \rightarrow A_{x_{i}+x_{i^{\prime}}, v_{j}+v_{j^{\prime}}}^{\prime}$ for $x_{i}, x_{i^{\prime}} \in V$ and $v_{j}, v_{j^{\prime}} \in F$.

\footnotetext{
${ }^{3}$ c.f. the last paragraph of page 384 and page 385 of Bana92.
} 
(4) $\neg\left(A_{x_{i}, v_{j}}^{\prime} \wedge A_{x_{i}, v_{l}}^{\prime}\right)$ for any $v_{l}, v_{j} \in F$ such that $v_{l} \neq v_{j}$ and each $x_{i} \in V$.

(5) $A_{x_{i}, v_{1}}^{\prime} \vee A_{x_{i}, v_{2}}^{\prime} \cdots \vee A_{x_{i}, v_{k}}^{\prime}$ for each $x_{i} \in V$.

By our assumption $V$ is well-orderable and $F$ is finite. So $F$ is well-orderable. Consequently, $V \times F$ is well-orderable. We enumerate $\operatorname{Var}=\left\{A_{x_{i}, v_{j}}^{\prime}: x_{i} \in V, v_{j} \in F\right\}$. Fix $V^{\prime} \in[V]^{<\omega}$. Let $W$ be the subspace of $V$ generated by the finite set $V^{\prime} \cup\{a\}$. We can see that $W$ is finite since $F$ is finite. Consequently, a linear functional $f: W \rightarrow F$ with $f(a)=1$ can be constructed in ZF. Following the methods used in the proof of Observation 3.2, we can obtain a non-zero linear functional $f: V \rightarrow F$.

Observation 3.7. For every $3 \leq k<\omega$, the statement $\mathcal{P}_{k}$ for the graph $G_{1}$ on some wellorderable set of vertices is provable under ZF.

Proof. Fix $3 \leq k<\omega$. Suppose $\chi\left(E_{G_{1}}\right)=k, \chi\left(E_{G_{2}}\right) \geq \omega$ and $G_{1}$ is a graph on some wellorderable set of vertices. First we observe that if $g: V_{G_{1}} \rightarrow\{1, \ldots, k\}$ is a good $k$-coloring of $G_{1}$, then $G(\langle x, y\rangle)=g(x)$ is a good $k$-coloring of $G_{1} \times G_{2}$. So, $\chi\left(E_{G_{1} \times G_{2}}\right) \leq k$. For the sake of contradiction assume that $F: V_{G_{1}} \times V_{G_{2}} \rightarrow\{1, \ldots, k-1\}$ is a good coloring of $G_{1} \times G_{2}$. For each color $c \in\{1, \ldots, k-1\}$ and each vertex $x \in V_{G_{1}}$ we let $A_{x, c}=\left\{y \in V_{G_{2}}: F(x, y)=c\right\}$.

claim 3.8. (ZF). For all finite $F \subset V_{G_{1}}$, there exists a mapping $i_{F}: F \rightarrow\{1, \ldots, k-1\}$ such that for any $x, x^{\prime} \in F, A_{x, i_{F}(x)} \cap A_{x^{\prime}, i_{F}\left(x^{\prime}\right)}$ is not independent.

Proof. Since any superset of non-independent set is non-independent, it is enough to show that for all finite $F \subset V_{G_{1}}$, there exists an $i_{F}: F \rightarrow\{1, \ldots, k-1\}$ such that $\cap_{x \in F} A_{x, i_{F}(x)}$ is not independent. For the sake of contradiction assume that there exist a finite $F \subset V_{G_{1}}$ such that for all $i_{F}: F \rightarrow\{1, \ldots, k-1\}, \cap_{x \in F} A_{x, i_{F}(x)}$ is independent. Now, $V_{G_{2}}=\cup_{i_{F}: F \rightarrow\{1, \ldots, k-1\}} \cap_{x \in F}$ $A_{x, i_{F}(x)}$. Thus $V_{G_{2}}$ can be written as a finite union of independent sets which contradicts the fact that $\chi\left(E_{G_{2}}\right)$ is infinite. Thus for all finite $F \subset V_{G_{1}}$, we can obtain a mapping $i_{F}: F \rightarrow$ $\{1, \ldots, k-1\}$ such that $\cap_{x \in F} A_{x, i_{F}(x)}$ is not independent.

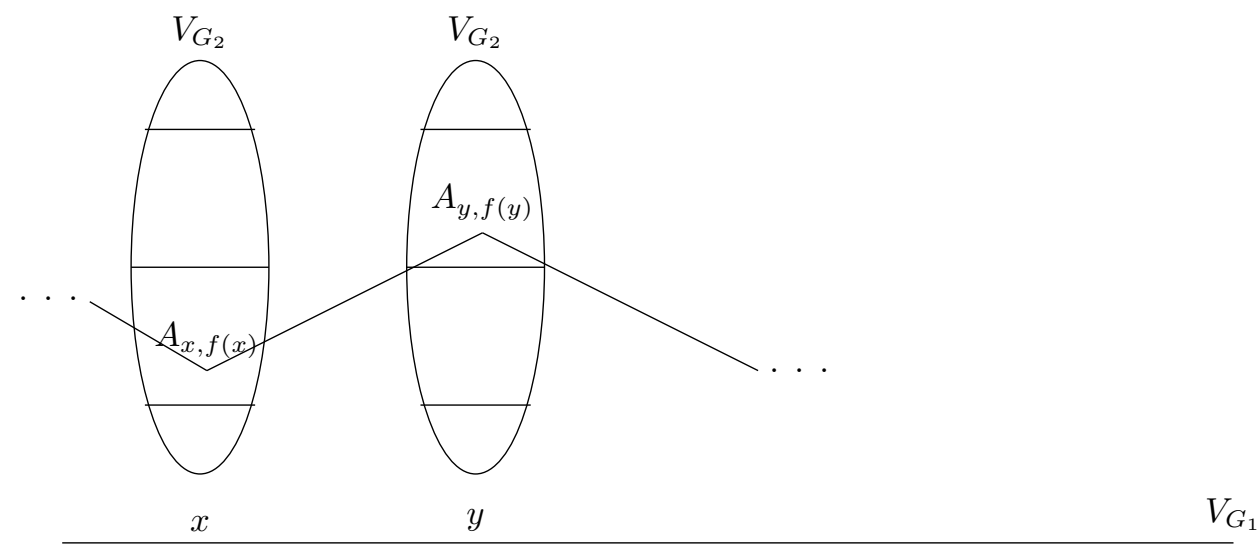

Figure 2. A map $f: V_{G_{1}} \rightarrow\{1, \ldots, k-1\}$ such that intersection of any two elements in $\left\{A_{x, f(x)}: x \in V_{G_{1}}\right\}$ is not independent.

Endow $\{1,2, \ldots, k-1\}$ with the discrete topology. Since $V_{G_{1}}$ is well-orderable, $\{1,2, \ldots, k-1\} \times V_{G_{1}}$ is well-orderable under ZF. Applying Theorem 1 of [Loeb65], $\{1,2, \ldots, k-1\}^{V_{G_{1}}}$ is compact. For $s \in\left[V_{G_{1}}\right]^{<\omega}$, define $F_{s}=\left\{f \in\{1,2, \ldots, k-1\}^{V_{G_{1}}}: x, y \in s, x \neq y \rightarrow A_{x, f(x)} \cap A_{y, f(y)}\right.$ is not independent $\}$. By claim 3.8, for each $s \in\left[V_{G_{1}}\right]^{<\omega}$ we have that $F_{s}$ is non-empty. We can see that $\left\{F_{s}: s \in\left[V_{G_{1}}\right]^{<\omega}\right\}$ has finite intersection property as $F_{s_{0} \cup \ldots \cup s_{k}} \subseteq F_{s_{0}} \cap \ldots \cap F_{s_{k}}$. Thus by compactness of $\{1,2, \ldots, k-1\}^{V_{G_{1}}}$, there is a $f \in \bigcap\left\{F_{s}: s \in\left[V_{G_{1}}\right]<\omega\right\}$. Clearly, for any $x, x^{\prime} \in V_{G_{1}}, A_{x, f(x)} \cap A_{x^{\prime}, f\left(x^{\prime}\right)}$ is not independent (see figure 2). Since $x \rightarrow f(x)$ is not a good coloring in $G_{1}$ as $\chi\left(E_{G_{1}}\right)=k$, there are $x, x^{\prime} \in V_{G_{1}}$ with $f(x)=f\left(x^{\prime}\right)=j$ and $\left\{x, x^{\prime}\right\} \in E_{G_{1}}$. 
Consequently, $A^{\prime}=A_{x, f(x)} \cap A_{x^{\prime}, f\left(x^{\prime}\right)}$ is not independent. Pick $y, y^{\prime} \in A^{\prime}$ joined by an edge in $E_{G_{2}}$. Then $(x, y)$ and $\left(x^{\prime}, y^{\prime}\right)$ are joined in $E_{G_{1}} \times E_{G_{2}}$ and get the same color $j$ which is a contradiction to the fact that $F$ is a good coloring of $G_{1} \times G_{2}$.

3.2. On partially ordered sets based on a well-ordered set of elements. The first author modifies the arguments from Claim 5 of [Tac16] and observes the following.

Observation 3.9. The following holds.

(1) $U T\left(\aleph_{0}, \aleph_{0}, \aleph_{0}\right)$ implies 'If in a partially ordered set based on a well-ordered set of elements, all chains are finite and all antichains are countable, then the set is countable'.

(2) $U T\left(\aleph_{\alpha}, \aleph_{\alpha}, \aleph_{\alpha}\right)$ implies 'If in a partially ordered set based on a well-ordered set of elements, all chains are finite and all antichains have size $\aleph_{\alpha}$, then the set has size $\aleph_{\alpha}$ ' for any regular $\aleph_{\alpha}$.

Proof. We prove Observation 3.9 (1). For the sake of contradiction, assume that all antichains of $(P, \leq)$ are countable, all chains of $(P, \leq)$ are finite, but the set $P$ is uncountable and wellordered. We construct an infinite chain in $(P, \leq)$ using $U T\left(\aleph_{0}, \aleph_{0}, \aleph_{0}\right)$ and obtain the desired contradiction.

claim 3.10. $\leq$ is a well-founded relation on $P$ i.e., every non-empty subset of $P$ has $a \leq-$ minimal element.

Proof. Let $P$ is well-orderable, say by $\preceq$. We claim that $\leq$ is a well-founded relation on $P$. Otherwise, there is a nonempty subset $P_{1} \subseteq P$ with no minimal elements. Consequently, using the fact that $\preceq$ is a well-ordering in $P$, we can obtain a strictly $\leq$-decreasing sequence of elements of $P_{1}$. This contradicts the assumption that $P$ has no infinite chains.

Without loss of generality we may assume $P=\cup\left\{P_{\alpha}: \alpha<\kappa\right\}$ where $\kappa$ is a well-ordered cardinal, $P_{0}$ is the set of minimal elements of $P$ and for each $\alpha<\kappa, P_{\alpha}$ is the set of minimal elements of $P \backslash \cup\left\{P_{\beta}: \beta<\alpha\right\}$. For each $\alpha<\kappa, P_{\alpha}$ is countable since $P_{\alpha}$ is an antichain.

- We note that $P=\cup\left\{P_{p}: p \in P_{0}\right\}$ where $P_{p}=\{q \in P: p \leq q\}$. Since $P$ is uncountable and $P_{0}$ is countable, $P_{p}$ is uncountable for some $p \in P_{0}$. Otherwise for all $p \in P_{0}, P_{p}$ is either countable or finite and $U T\left(\aleph_{0}, \aleph_{0}, \aleph_{0}\right)+U T\left(\aleph_{0}\right.$, fin, $\left.\aleph_{0}\right)$ implies $P$ is countable which is a contradiction. Now $U T\left(\aleph_{0}, \aleph_{0}, \aleph_{0}\right)$ implies $U T\left(\aleph_{0}, f i n, \aleph_{0}\right)$ in ZF, thus $U T\left(\aleph_{0}, \aleph_{0}, \aleph_{0}\right)$ suffices. Since $\left\{q \in P_{0}: P_{q}\right.$ is uncountable $\}$ is a non-empty subset of $P$, we can find a least $p_{0} \in P_{0}$ with respect to $\preceq$ such that $P_{p_{0}}$ is uncountable.

- Let us consider $P^{\prime}=P_{p_{0}} \backslash\left\{p_{0}\right\}$. So, $P^{\prime}$ is uncountable. Again if $P_{1}^{\prime}$ is the set of minimal elements of $P^{\prime}$, we can write $P^{\prime}=\cup\left\{P_{p}: p \in P_{1}^{\prime}\right\}$ where $P_{p}=\{q \in P: p \leq q\}$. Since $P^{\prime}$ is uncountable and $P_{1}^{\prime}$ is countable (since all antichains of $(P, \leq)$ are countable by assumption), once again applying $U T\left(\aleph_{0}, \aleph_{0}, \aleph_{0}\right)$ as in the previous paragraph, $P_{p}$ is uncountable for some $p \in P_{1}^{\prime}$. Since $\left\{q \in P_{1}^{\prime}: P_{q}\right.$ is uncountable $\}$ is a non-empty subset of $P$, we can find a least $p_{1} \in P_{1}^{\prime}$ with respect to $\preceq$ such that $P_{p_{1}}$ is uncountable. We can see that $p_{0}<p_{1}$.

Continuing this process step by step we obtain a sequence $\left\langle p_{n}: n \in \omega\right\rangle$ of elements of $P$ such that $p_{n}<p_{n+1}$ for each $n \in \omega$. Consequently, we obtain an infinite chain.

Remark. Similarly for any regular $\aleph_{\alpha}$, assuming $U T\left(\aleph_{\alpha}, \aleph_{\alpha}, \aleph_{\alpha}\right)$ we can prove the following since alephs are well-ordered. 'If in a partially ordered set based on well-ordered set of elements, all chains are finite and all antichains have size $\aleph_{\alpha}$, then the set has size $\aleph_{\alpha}$.' Consequently, we can prove Observation 3.9(2).

\section{Consistency Results}

Theorem 4.1. For every natural number $n \geq 2$, there is a permutation model $\mathcal{N}$ of $Z F A$ where $C A C$ holds and $A C_{n}^{-}$fails. Moreover, we can observe the following in the model. 
(1) CS, as well as CWF, holds.

(2) DT holds, $M A\left(\aleph_{0}\right)$ fails, MCC fails, LT fails.

(3) If in a partially ordered set, all chains are finite and all antichains are countable, then the set is countable. Moreover, if in a partially ordered set, all chains are finite and all antichains have size $\aleph_{\alpha}$, then the set has size $\aleph_{\alpha}$ for any regular $\aleph_{\alpha}$.

(4) The following statements hold.

(a) Marshall Hall's theorem for linearly-ordered collection of finite subsets of a set.

(b) For every $3 \leq k<\omega, \mathcal{P}_{k}$ holds for any graph $G_{1}$ on some linearly-orderable set of vertices.

(c) For an infinite graph $G=\left(V_{G}, E_{G}\right)$ on a linearly-ordered set of vertices $V_{G}$ and a finite graph $H=\left(V_{H}, E_{H}\right)$, if every finite subgraph of $G$ has a homomorphism into $H$, then so has $G$.

(d) For every finite field $\mathcal{F}=\langle F, \ldots\rangle$, for every nontrivial linearly-orderable vector space $V$ over $\mathcal{F}$, there exists a non-zero linear functional $f: V \rightarrow F$.

Proof. In Theorem 8 of [HT19], Halbeisen and Tachtsis constructed a permutation model $\mathcal{N}$ where for arbitrary $n \geq 2, A C_{n}^{-}$fails but CAC holds. We fix an arbitrary integer $n \geq 2$ and recall the model constructed in the proof of Theorem 8 of [HT19] as follows.

- Defining the ground model $M$. We start with a ground model $M$ of $Z F A+A C$ where $A$ is a countably infinite set of atoms written as a disjoint union $\cup\left\{A_{i}: i \in \omega\right\}$ where for each $i \in \omega, A_{i}=\left\{a_{i_{1}}, a_{i_{2}}, \ldots a_{i_{n}}\right\}$.

- Defining the group $\mathcal{G}$ of permutations and the filter $\mathcal{F}$ of subgroups of $\mathcal{G}$.

- Defining $\mathcal{G} . \mathcal{G}$ is defined in HT19 in a way so that if $\eta \in \mathcal{G}$, then $\eta$ only moves finitely many atoms and for all $i \in \omega, \eta\left(A_{i}\right)=A_{k}$ for some $k \in \omega$. We recall the details from [HT19] as follows. For all $i \in \omega$, let $\tau_{i}$ be the $n$-cycle $a_{i_{1}} \mapsto a_{i_{2}} \mapsto \ldots a_{i_{n}} \mapsto a_{i_{1}}$. For every permutation $\psi$ of $\omega$, which moves only finitely many natural numbers, let $\phi_{\psi}$ be the permutation of $A$ defined by $\phi_{\psi}\left(a_{i_{j}}\right)=a_{\psi(i)_{j}}$ for all $i \in \omega$ and $j=1,2, \ldots, n$. Let $\eta \in \mathcal{G}$ if and only if $\eta=\rho \phi_{\psi}$ where $\psi$ is a permutation of $\omega$ which moves only finitely many natural numbers and $\rho$ is a permutation of $A$ for which there is a finite $F \subseteq \omega$ such that for every $k \in F$, $\rho \uparrow A_{k}=\tau_{k}^{j}$ for some $j<n$, and $\rho$ fixes $A_{m}$ pointwise for every $m \in \omega \backslash F$.

- Defining $\mathcal{F}$. Let $\mathcal{F}$ be the filter of subgroups of $\mathcal{G}$ generated by $\left\{\operatorname{fix}_{\mathcal{G}}(E): E \in\right.$ $[A]<\omega\}$.

- Defining the permutation model. Consider the permutation model $\mathcal{N}$ determined by $M, \mathcal{G}$ and $\mathcal{F}$.

Following point 1 in the proof of Theorem 8 of [HT19], both $A$ and $\mathcal{A}=\left\{A_{i}\right\}_{i \in \omega}$ are amorphous in $\mathcal{N}$ and no infinite subfamily $\mathcal{B}$ of $\mathcal{A}$ has a Kinna-Wegner selection function. Consequently, $A C_{n}^{-}$fails. The first author observes the following.

Lemma 4.2. In $\mathcal{N}, D T, C S$ as well as $C W F$ holds. Moreover the following holds in $\mathcal{N}$.

- If in a partially ordered set, all chains are finite and all antichains are countable, then the set is countable.

- 'If in a partially ordered set, all chains are finite and all antichains have size $\aleph_{\alpha}$, then the set has size $\aleph_{\alpha}^{\prime}$ for any regular $\aleph_{\alpha}$.

Proof. We follow the steps below.

(1) Let $(P, \leq)$ be a p.o.set in $\mathcal{N}$ and $E \in[A]^{<\omega}$ be a support of $(P, \leq)$. We can write $P$ as a disjoint union of $\mathrm{x}_{\mathcal{G}}(E)$-orbits, i.e., $P=\bigcup\left\{\operatorname{Orb}_{E}(p): p \in P\right\}$, where $\operatorname{Orb}_{E}(p)=\{\phi(p)$ : $\left.\phi \in \mathrm{x}_{\mathcal{G}}(E)\right\}$ for all $p \in P$. The family $\left\{\operatorname{Orb}_{E}(p): p \in P\right\}$ is well-orderable in $\mathcal{N}$ since $\mathrm{x}_{\mathcal{G}}(E) \subseteq \operatorname{Sym}_{\mathcal{G}}\left(\operatorname{Orb}_{E}(p)\right)$ for all $p \in P$.

(2) Since if $\eta \in \mathcal{G}$, then $\eta$ only moves finitely many atoms, $\operatorname{Orb}_{E}(p)$ is an antichain in $P$ for each $p \in P$. Otherwise there is a $p \in P$, such that $\operatorname{Orb}_{E}(p)$ is not an antichain in $(P, \leq)$. Thus, for some $\phi, \psi \in \operatorname{fix}_{\mathcal{G}}(E), \phi(p)$ and $\psi(p)$ are comparable. Without loss of 
generality we may assume $\phi(p)<\psi(p)$. Since if $\eta \in \mathcal{G}$, then $\eta$ only moves finitely many atoms, there exists some $k<\omega$ such that $\phi^{k}=1_{A}$. Let $\pi=\psi^{-1} \phi$. Consequently, $\pi(p)<p$ and $\pi^{k}=1_{A}$ for some $k \in \omega$. Thus, $p=\pi^{k}(p)<\pi^{k-1}(p)<\ldots<\pi(p)<p$. By transitivity of $<, p<p$, which is a contradiction.

(3) We prove that in $\mathcal{N}, D T$ holds. Let $E \subset A$ be a finite support of an infinite p.o.set $\mathbb{P}=(P,<)$ with finite width. Then $P=\bigcup\left\{\operatorname{Orb}_{E}(p): p \in P\right\}$. Following $(2), \operatorname{Orb}_{E}(p)$ is an antichain in $\mathbb{P}$. Consequently, $\operatorname{Orb}_{E}(p)$ is finite for each $p \in P$ since the width of $\mathbb{P}$ is finite. Following $(1),\left\{O r b_{E}(p): p \in P\right\}$ is well-orderable in $\mathcal{N}$. Following point 4 in the proof of Theorem 8 of [HT19] and Lemma 3 of [Tac16], UT(WO,WO,WO) holds in $\mathcal{N}$, and so $P$ is well-orderable in $\mathcal{N}$. Applying Theorem 3.1(i) of Tac19], DT holds in $\mathcal{N}$.

(4) To see that CS as well as CWF holds in $\mathcal{N}$ we follow Theorem 3.26 of [THS16] and Theorem 10(ii) of Tac17 respectively. We sketch the important steps below.

(a) We follow Theorem $\mathbf{3 . 2 6}$ of [THS16] to see that CS holds in $\mathcal{N}$ as follows. Let $(P, \leq)$ be a poset without maximal elements supported by E. Following $(1), \mathcal{O}=$ $\left\{\operatorname{Orb}_{E}(p): p \in P\right\}$ is a well-ordered partition of $P$. Define $\preceq$ on $\mathcal{O}$, as $X \preceq Y \leftrightarrow$ $\exists x \in X, \exists y \in Y$ such that $x \leq y$. Since $(P, \leq)$ has no maximal element, $(\mathcal{O}, \preceq)$ has no maximal element following (2). Since $\mathcal{O}$ is well-ordered there exists a partition $\mathcal{U}_{\mathcal{O}}=\{\mathcal{Q}, \mathcal{R}\}$ of $\mathcal{O}$ in 2 cofinal subsets. Consequently, $\mathcal{U}_{P}=\{\cup \mathcal{Q}, \cup \mathcal{R}\}$ is a partition of $P$ in 2 cofinal subsets.

(b) We follow Theorem 10 (ii) of [Tac17] to see that CWF holds in $\mathcal{N}$ as follows. Let $(P, \leq)$ be a poset supported by $\mathcal{N}$. Since $\mathcal{O}=\left\{\operatorname{Orb}_{E}(p): p \in P\right\}$ is well-orderable, it has a cofinal well-founded subset $\mathcal{W}=\left\{W_{\alpha}: \alpha<\gamma\right\}$ such that for $\beta<\alpha, W_{\alpha} \npreceq W_{\beta}$ for all $\beta, \alpha<\gamma$. Consequently, $C=\cup \mathcal{W}$ is a cofinal well-founded subset of $P$.

(5) We show the following in $\mathcal{N}$.

'If in a partially ordered set, all chains are finite and all antichains are countable, then the set is countable.'

It is known that in every $F M$-model $U T(W O, W O, W O)$ implies $(\forall \alpha) U T\left(\aleph_{\alpha}, \aleph_{\alpha}, \aleph_{\alpha}\right)$ (c.f. page 176 of [HR98]). Consequently, $U T\left(\aleph_{0}, \aleph_{0}, \aleph_{0}\right)$ holds in $\mathcal{N}$. Let $(P,<)$ be an uncountable p.o.set in $\mathcal{N}$ where all antichains are countable and $E \in[A]^{<\omega}$ be a support of $(P,<)$. Following $(1), \mathcal{O}=\left\{\operatorname{Orb}_{E}(p): p \in P\right\}$ is a well-ordered partition of $P$ since for all $p \in P, E$ is a support of $\operatorname{Orb}_{E}(p)$. Following $(2), \operatorname{Orb}_{E}(p)$ is an antichain and hence countable. Consequently, $\operatorname{Orb}_{E}(p)$ is well-orderable. Since $U T(W O, W O, W O)$ holds in $\mathcal{N}, P$ is well-orderable. By Observation 3.9(1), since $U T\left(\aleph_{0}, \aleph_{0}, \aleph_{0}\right)$ holds in $\mathcal{N}$, there is an infinite chain in $\mathcal{N}$.

(6) Following (5) and Observation 3.9(2), we can prove the following in $\mathcal{N}$.

'If in a partially ordered set $(P,<)$, all chains are finite and all antichains have size $\aleph_{\alpha}$, then the set has size $\aleph_{\alpha}$ '.

Remark. The referee pointed out that the statements If in a partially ordered set based on a well-ordered set of elements all chains are finite and all antichains are countable then the set is countable and If in a partially ordered set based on a well-ordered set of elements all chains are finite and all antichains have size $\aleph_{\alpha}$ then the set has size $\aleph_{\alpha}$ are true in all Fraenkel-Mostowski permutation models. So Observation 3.9(1) and Observation 3.9(2) are not needed in the proofs of parts (5) and (6) of the proof of Lemma 4.2 .

Lemma 4.3. In $\mathcal{N}, M A\left(\aleph_{0}\right)$ fails.

Proof. Since $A$ is amorphous, the statement 'for all infinite $X, 2^{X}$ is Baire' is false following Remark 2.7 of Tac16b]. Since CAC holds in $\mathcal{N}, A C_{\text {fin }}^{\omega}$ holds as well (c.f. Lemma 4.4 of Tac19a] $)$. Consequently, $M A\left(\aleph_{0}\right)$ fails following Remark 2.7 of [Tac16b].

Lemma 4.4. In $\mathcal{N}, M C C$ fails. 
Proof. Modifying the proof of Theorem 8 (ii) of Tac17, we can see that $n(A)=n$. Thus there is a bounded amorphous set A. Consequently, MCC fails by Theorem 6 of Tac17.

Lemma 4.5. In $\mathcal{N}$, LT fails.

Proof. Since $\mathcal{A}$ is an amorphous set of non-empty sets which has no choice function in $\mathcal{N}$, following Lemma 4.1(i) Tac19a, LT fails in $\mathcal{N}$.

Lemma 4.6. In $\mathcal{N}$, the following statements hold for linearly-ordered structures.

(1) Marshall Hall's theorem for linearly-ordered collection of finite subsets of a set.

(2) For every $3 \leq k<\omega, \mathcal{P}_{k}$ holds for any graph $G_{1}$ on some linearly-orderable set of vertices.

(3) For an infinite graph $G=\left(V_{G}, E_{G}\right)$ on a linearly-ordered set of vertices $V_{G}$ and a finite graph $H=\left(V_{H}, E_{H}\right)$, if every finite subgraph of $G$ has a homomorphism into $H$, then so has $G$.

(4) For every finite field $\mathcal{F}=\langle F, \ldots\rangle$, for every nontrivial linearly-orderable vector space $V$ over $\mathcal{F}$, there exists a non-zero linear functional $f: V \rightarrow F$.

Proof. Since $U T(W O, W O, W O)$ and LW holds in $\mathcal{N}$ (c.f. pt 4 and pt 3 in the proof of Theorem 8 in [HT19]), (1), (2), (3) and (4) hold in $\mathcal{N}$ following the observations in section 3.

Remark 1. In Theorem 7 of Tac19a, Tachtsis generalized the above construction and proved that $A C^{L O}+L W \nrightarrow L L T$ by constructing a permutation model $\mathcal{N}$. Since $A C^{W O}$ holds in $\mathcal{N}$, $D C$ holds in $\mathcal{N}$ as well (c.f. Theorem $\mathbf{8 . 2}$ of Jec73). We observe another standard argument to see that $D C$ holds in $\mathcal{N}$. Since $\mathcal{I}$ is closed under countable unions in the model, we can see that DC holds in $\mathcal{N}$. Let $\mathcal{R}$ is a relation in $\mathcal{N}$ such that if $x \in \operatorname{dom}(\mathcal{R})$, there exists a $y$ such that $x R y$. Consequently, there is a sequence $\left\langle x_{n}: n \in \omega\right\rangle$ in the ground model $M$ such that for each $n \in \omega, x_{n} R x_{n+1}$. If $x_{n}$ is supported by $E_{n}$ for every $n \in \omega$, then $\left\langle x_{n}: n \in \omega\right\rangle$ is supported by $\cup_{n \in \omega} E_{n}$. Since $\mathcal{I}$ is closed under countable unions, the sequence $\left\langle x_{n}: n \in \omega\right\rangle$ is in $\mathcal{N}$.

A class of models $M_{\aleph_{\alpha}}$ for any regular cardinal $\aleph_{\alpha}$ (similar to the model $M_{\aleph_{1}}$ constructed in Theorem 7 of (Tac19a] ) can be defined where $A C^{L O}$ and $L W$ holds but $L T$ fails, by replacing $\aleph_{1}$ by $\aleph_{\alpha}$. Moreover in $M_{\aleph_{\alpha}}, D C_{<\aleph_{\alpha}}$ holds since $\mathcal{I}$ is closed under $<\aleph_{\alpha}$ unions.

Remark 2. In the permutation model $\mathcal{N}$ of [Tac16], CS, as well as CWF, holds following the work in this section. Moreover, the following statement holds in $\mathcal{N}$, following the work in this section.

'If in a partially ordered set, all chains are finite and all antichains are countable, then the set is countable.'

Theorem 4.7. There is a permutation model $\mathcal{N}$ of $Z F A$, where there is an amorphous set. Moreover, the following holds in $\mathcal{N}$.

(1) If in a partially ordered set, all chains are finite and all antichains are countable, then the set is countable.

(2) If in a partially ordered set, all chains are finite and all antichains have size $\aleph_{\alpha}$, then the set has size $\aleph_{\alpha}$ for any regular $\aleph_{\alpha}$.

Proof. We consider the basic Fraenkel model (labeled as Model $\mathcal{N}_{1}$ in HR98) where 'there are no amorphous sets' is false and $U T(W O, W O, W O)$ holds (c.f. [HR98]). Let $(P, \leq)$ be a p.o.set in $\mathcal{N}_{1}$, and $E$ be a nite support of $(P, \leq)$. By (1) in the proof of Lemma 4.2, $\mathcal{O}=\left\{\operatorname{Orb}_{E}(p): p \in P\right\}$ is a well-ordered partition of $P$. Now for each $p \in P, \operatorname{Orb}_{E}(p)$ is an antichain (c.f. the proof of Lemma 9.3 in [Jec73]). Thus, by methods of Lemma 4.2, (1) and (2) hold in $\mathcal{N}_{1}$. 
Theorem 4.8. There is a permutation model of ZFA where CS, as well as CWF, holds, but $A C_{\text {fin }}^{\omega}$ fails. Moreover, the following statements hold in the model.

(1) For every $3 \leq k<\omega, \mathcal{P}_{k}$ holds for any graph $G_{1}$ on some linearly-orderable set of vertices.

(2) For an infinite graph $G=\left(V_{G}, E_{G}\right)$ on a linearly-ordered set of vertices $V_{G}$ and a finite graph $H=\left(V_{H}, E_{H}\right)$, if every finite subgraph of $G$ has a homomorphism into $H$, then so has $G$.

(3) For every finite field $\mathcal{F}=\langle F, \ldots\rangle$, for every nontrivial linearly-orderable vector space $V$ over $\mathcal{F}$, there exists a non-zero linear functional $f: V \rightarrow F$.

Proof. We recall the Lévy's permutation model (labeled as Model $\mathcal{N}_{6}$ in [HR98]).

- Defining the ground model $M$. We start with a ground model $M$ of $Z F A+A C$ where $A$ is a countably infinite set of atoms written as a disjoint union $\cup\left\{P_{n}: n \in \omega\right\}$, where $P_{n}=\left\{a_{1}^{n}, \ldots a_{p_{n}}^{n}\right\}$ such that $p_{n}$ is the $n^{\text {th }}$-prime number.

- Defining the group $\mathcal{G}$ of permutations and the filter $\mathcal{F}$ of subgroups of $\mathcal{G}$.

- Defining $\mathcal{G}$. $\mathcal{G}$ be the group generated by the following permutations $\pi_{n}$ of $A$.

$$
\pi_{n}: a_{1}^{n} \mapsto a_{2}^{n} \mapsto \ldots a_{p_{n}}^{n} \mapsto a_{1}^{n} \text { and } \pi_{n}(x)=x \text { for all } x \in A \backslash P_{n} .
$$

- Defining $\mathcal{F} . \mathcal{F}$ be the filter of subgroups of $\mathcal{G}$ generated by $\left\{\right.$ fix $\left._{\mathcal{G}}(E): E \in[A]^{<\omega}\right\}$.

- Defining the permutation model. Consider the permutation model $\mathcal{N}_{6}$ determined by $M, \mathcal{G}$ and $\mathcal{F}$.

It is well-known that in $\mathcal{N}_{6}, A C_{\text {fin }}^{\omega}$ fails since $\left\{P_{i}: i \in \omega\right\}$ has no (partial) choice function (c.f. Jec73]). Consequently, following Lemma 4.4 of [Tac19a, CAC fails in $\mathcal{N}_{6}$. Since every permutation $\phi \in \mathcal{G}$ moves only finitely many atoms, following the arguments in Lemma 4.2, we can observe that CS, as well as CWF, holds in $\mathcal{N}_{6}$.

Lemma 4.9. In $\mathcal{N}_{6}, L W$ holds.

Proof. Let $(X, \leq)$ be a linearly ordered set in $\mathcal{N}_{6}$ supported by E. We show fix $\mathcal{G} E \subseteq$ fix $_{\mathcal{G}} X$ which implies that $X$ is well-orderable in $\mathcal{N}_{6}$. For the sake of contrary assume fix $\operatorname{G}_{\mathcal{G}} E \nsubseteq \operatorname{fix}_{\mathcal{G}} X$. So there is an element $y \in X$ which is not supported by $E$ and there is a $\phi \in$ fix $_{\mathcal{G}} E$ such that $\phi(y) \neq y$. Since $\phi(y) \neq y$ and $\leq$ is a linear order on $X$, we obtain either $\phi(y)<y$ or $y<\phi(y)$. Let $\phi(y)<y$. Since every permutation $\phi \in \mathcal{G}$ moves only finitely many atoms there exists some $k<\omega$ such that $\phi^{k}=1_{A}$. Thus, $p=\phi^{k}(p)<\phi^{k-1}(p)<\ldots<\phi(p)<p$ which is a contradiction. Similarly we can arrive at a contradiction if we assume $y<\phi(y)$.

Since LW holds in $\mathcal{N}_{6}$, we can observe (1), (2) and (3) in $\mathcal{N}_{6}$ by observations in section 3.

Theorem 4.10. There is a permutation model of $Z F A$ where CS, as well as CWF, holds, but LOKW $W_{4}^{-}$fails. Moreover, the following statements hold in the model.

(1) For every $3 \leq k<\omega, \mathcal{P}_{k}$ holds for any graph $G_{1}$ on some linearly-orderable set of vertices.

(2) For an infinite graph $G=\left(V_{G}, E_{G}\right)$ on a linearly-ordered set of vertices $V_{G}$ and a finite graph $H=\left(V_{H}, E_{H}\right)$, if every finite subgraph of $G$ has a homomorphism into $H$, then so has $G$.

(3) For every finite field $\mathcal{F}=\langle F, \ldots\rangle$, for every nontrivial linearly-orderable vector space $V$ over $\mathcal{F}$, there exists a non-zero linear functional $f: V \rightarrow F$.

Proof. We recall the permutation model $\mathcal{M}$ from the second assertion of Theorem 10(ii) of HT19.

- Defining the ground model $M$. Let $\kappa$ be any infinite well-ordered cardinal number. We start with a ground model $M$ of $Z F A+A C$ where $A$ is a $\kappa$-sized set of atoms written as a disjoint union $\cup\left\{A_{\alpha}: \alpha<\kappa\right\}$, where $A_{\alpha}=\left\{a_{\alpha, 1}, a_{\alpha, 2}, a_{\alpha, 3}, a_{\alpha, 4}\right\}$ such that $\left|A_{\alpha}\right|=4$ for all $\alpha<\kappa$. 
- Defining the group $\mathcal{G}$ of permutations and the filter $\mathcal{F}$ of subgroups of $\mathcal{G}$.

- Defining $\mathcal{G}$. Let $\mathcal{G}$ be the weak direct product of $\mathcal{G}_{\alpha}$ 's where $\mathcal{G}_{\alpha}$ is the alternating group on $\mathcal{A}_{\alpha}$ for each $\alpha<\kappa$.

- Defining $\mathcal{F}$. Let $\mathcal{F}$ be the normal filter of subgroups of $\mathcal{G}$ generated by $\left\{\operatorname{fix}_{\mathcal{G}}(E)\right.$ : $E \in[A]<\omega\}$.

- Defining the permutation model. Consider the permutation model $\mathcal{M}$ determined by $M, \mathcal{G}$ and $\mathcal{F}$.

In $\mathcal{M}, L O K W_{4}^{-}$fails (c.f. Theorem 10(ii) of [HT19]). Since every permutation, $\phi \in \mathcal{G}$ moves only finitely many atoms, following the arguments in Lemma $\mathbf{4 . 2}$ we can observe that CS, as well as CWF, holds in $\mathcal{M}$. Since LW holds in $\mathcal{M}$ (c.f. Theorem 10(ii) of [HT19]), we can observe (1), (2) and (3) in $\mathcal{M}$ by observations in section 3.

\section{Observations in Howard's model}

Theorem 5.1. For any $3 \leq k<\omega, \mathcal{P}_{k}$ follows from $F_{k-1}$ in $Z F$. Moreover, if $X \in\left\{A C_{3}, A C_{\text {fin }}^{\omega}\right\}$, then the statement $\mathcal{P}_{k}$ does not imply $X$ in $Z F A$ when $k=3$.

Proof. Fix $3 \leq k<\omega$. Suppose $\chi\left(E_{G_{1}}\right)=k, \chi\left(E_{G_{2}}\right) \geq \omega$ and $G_{1}$ is a graph on some wellorderable set of vertices. First we observe that if $g: V_{G_{1}} \rightarrow\{1, \ldots, k\}$ is a good $k$-coloring of $G_{1}$, then $G(\langle x, y\rangle)=g(x)$ is a good $k$-coloring of $G_{1} \times G_{2}$. So, $\chi\left(E_{G_{1} \times G_{2}}\right) \leq k$. For the sake of contradiction assume that $F: V_{G_{1}} \times V_{G_{2}} \rightarrow\{1, \ldots, k-1\}$ is a good coloring of $G_{1} \times G_{2}$. For each color $c \in\{1, \ldots, k-1\}$ and each vertex $x \in V_{G_{1}}$ we let $A_{x, c}=\left\{y \in V_{G_{2}}: F(x, y)=c\right\}$. Define a relation $R$ on $\{1, \ldots, k-1\}$ as $\left(v_{1}, i\right) R\left(v_{2}, j\right)$ if and only if ' $v_{1} \neq v_{2}$ implies $A_{v_{1}, i} \cap A_{v_{2}, j}$ is not independent' for $v_{1}, v_{2} \in V_{G_{1}}$. By $F_{k-1}$ and claim 3.8 there exist a choice function $f$ such that for any $x, x^{\prime} \in V_{G_{1}}, A_{x, f(x)} \cap A_{x^{\prime}, f\left(x^{\prime}\right)}$ is not independent. Since $x \rightarrow f(x)$ is not a good coloring in $G_{1}$ as $\chi\left(E_{G_{1}}\right)=k$, there are $x, x^{\prime} \in V_{G_{1}}$ with $f(x)=f\left(x^{\prime}\right)=j$ and $\left\{x, x^{\prime}\right\} \in E_{G_{1}}$. Consequently, $A^{\prime}=A_{x, f(x)} \cap A_{x^{\prime}, f\left(x^{\prime}\right)}$ is not independent. Pick $y, y^{\prime} \in A^{\prime}$ joined by an edge in $E_{G_{2}}$. Then $(x, y)$ and $\left(x^{\prime}, y^{\prime}\right)$ are joined in $E_{G_{1}} \times E_{G_{2}}$ and get the same color $j$ which is a contradiction to the fact that $F$ is a good coloring of $G_{1} \times G_{2}$.

For the second assertion, we consider the permutation model $\mathcal{N}$ from section 3 of How84] where $A C_{3}$ fails, and $F_{2}$ holds. Consequently, $\mathcal{P}_{3}$ holds in $\mathcal{N}$. In $\mathcal{N}$, there is a countable family $\mathcal{A}=\left\{A_{i}: i \in \omega\right\}$ which has no partial choice function. Consequently, $P A C_{\text {fin }}^{\omega}$ fails. Since $P A C_{\text {fin }}^{\omega}$ is equivalent to $A C_{\text {fin }}^{\omega}$ (see the proof of Lemma 4.4 of [Tac19a] $), A C_{\text {fin }}^{\omega}$ fails in $\mathcal{N}$.

Question 5.2. If $k>3$, does $U L$ follow from $\mathcal{P}_{k}$ ? Otherwise is there any model of ZF or ZFA, where $\mathcal{P}_{k}$ holds for $k>3$, but UL fails?

Theorem 5.3. For any $2 \leq k<\omega, \mathcal{P}_{G, H}$ restricted to finite graph $H$ with $k$ vertices follows from $F_{k}$ in $Z F$. Moreover, if $X \in\left\{A C_{3}, A C_{\text {fin }}^{\omega}\right\}$, then $\mathcal{P}_{G, H}$ restricted to finite graph $H$ with 2 vertices does not imply $X$ in $Z F A$.

Proof. Fix $2 \leq k<\omega$. Let $V_{H}=\left\{v_{1}, \ldots v_{k}\right\}$. For each $x \in V_{G}$, let $A_{x}=\left\{\left(x, v_{1}\right), \ldots\left(x, v_{k}\right)\right\}$. Define a relation $R$ on $\cup_{x \in V_{G}} A_{x}$ by $\left(x, v_{i}\right) R\left(x^{\prime}, v_{j}\right)$ if and only if ' $\left\{x, x^{\prime}\right\} \in E_{G}$ implies $\left\{v_{i}, v_{j}\right\} \in E_{H}$ ' for $\left(x, v_{i}\right) \in A_{x},\left(x^{\prime}, v_{j}\right) \in A_{x^{\prime}}$. By assumption, for all finite $F \subset V_{G}$, there exists a homomorphism $h_{F}: G \uparrow F \rightarrow H$. For any finite $F \subset V_{G}$, and an homomorphism $h_{F}$ of $F$, let $h_{F}{ }^{*}(j)=\left(j, h_{F}(j)\right)$ for $j \in F$. Clearly, $h_{F}{ }^{*}$ is an $R$-consistent choice function for $\left\{A_{x}\right\}_{x \in F}$. By $F_{k}$, there is a $R$-consistent choice function $h_{F}{ }^{*}$ for $\left\{A_{x}\right\}_{x \in V_{G}}$. Define $h_{V_{G}}$ on $V_{G}$ by $h_{V_{G}}{ }^{*}(j)=\left(j, h_{V_{G}}(j)\right)$ for $j \in V_{G}$. Let $\left(j, j^{\prime}\right) \in E_{G}$ such that $j, j^{\prime} \in V_{G}$. Since $i_{I}{ }^{*}$ is $R$-consistent, $\left(j, h_{V_{G}}(j)\right) R\left(j^{\prime}, h_{V_{G}}\left(j^{\prime}\right)\right)$. By the definition of $R,\left(h_{V_{G}}(j), h_{V_{G}}\left(j^{\prime}\right)\right) \in E_{H}$.

For the second assertion, we once more consider the permutation model $\mathcal{N}$ from section 3 of How84 where $A C_{3}$ and $A C_{\text {fin }}^{\omega}$ fails, and $F_{2}$ holds. Consequently, ' $\mathcal{P}_{G, H}$ for a finite graph $H$ with 2 vertices' holds in $\mathcal{N}$. 
Acknowledgement. We would like to thank the reviewer for reading the manuscript carefully and providing suggestions for improvement.

\section{REFERENCES}

[Bana92] B. Banaschewski, Algebraic closure without choice, MLQ Math. Log. Q. 38 (1992), no. 1, pp. 383-385.

[Cow77] R. H. Cowen, Generalizing König's infinity lemma, Notre Dame J. Formal Logic 18 (1977), no. 2, pp. 243-247.

[Dil50] R. P. Dilworth, A decomposition theorem for partially ordered sets, Ann. of Math. 51 (1950), pp. 161166.

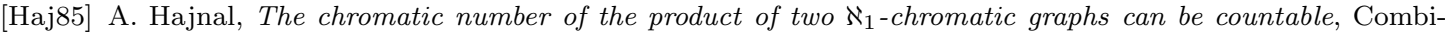
natorica 5 (1985), pp. 137140.

[Hal48] M. Hall Jr., Distinct representatives of subsets, Bull. Amer. Math. Soc. 54(1948), pp. 922-926.

[HR98] P. Howard and J. E. Rubin, Consequences of the Axiom of Choice, Mathematical Surveys and Monographs 59 (1998), DOI: http://dx.doi.org/10.1090/surv/059 MR 1637107

[HT19] L. Halbeisen and E. Tachtsis, On Ramsey Choice and Partial Choice for infinite families of n-element sets, Arch. Math. Logic (2019). DOI: https://doi.org/10.1007/s00153-019-00705-7.

[HT13] P. Howard and E. Tachtsis, On vector spaces over specific fields without choice, MLQ Math. Log. Q. 59 (2013), no. 3

[How84] P. Howard, Binary consistent choice on pairs and a generalization of Konig's infinity lemma, Fund. Math. 121 (1984), pp. 17-23.

[Jec73] T. J. Jech, The axiom of choice. North-Holland Publishing Co., Amsterdam, 1973, Studies in Logic and the Foundations of Mathematics, Vol. 75. MR 0396271

[Ker00] K. Keremedis, The compactness of $2^{\mathbb{R}}$ and the axiom of choice, MLQ Math. Log. Q. 46 (2000), pp. 569-571.

[KT06] P. Komjáth and V. Totik, Problems in Classical Set Theory, Springer (2006).

[Kom] P. Komjáth, Infinite graphs, Manuscript under preperation.

[Loeb65] P. Loeb, A New Proof of the Tychonoff Theorem, The American Mathematical Monthly 72 (1965), no. 7, pp. 711-717.

[LN51] J. Łoś and Czesław Ryll-Nardzewski, On the application of Tychonoff's theorem in mathematical proofs, Fund. Math. 38 (1951), no. 1, pp. 233-237. ISSN: 0016-2736.

[Tac19] E. Tachtsis, Dilworth's decomposition theorem for posets in ZF, Acta Math. Hungar. 159 (2019), pp. 603-617, DOI: 10.1007/s10474-019-00967-w.

[Tac19a] L Lośs theorem and the axiom of choice, MLQ Math. Log. Q. 65 (2019), no. 3, pp. 280-292, DOI: $10.1002 /$ malq.201700074.

[Tac17] — On the Minimal Cover Property and Certain Notions of Finite, Arch. Math. Logic. 57 (2018), no. 5-6, pp. 665-686.

[Tac17a] —. On variants of the principle of consistent choices, the minimal cover property and the 2compactness of generalized Cantor cubes, Topology and its Applications (Elsevier). 219 (2017), pp. 122-140.

[Tac16] — On Ramsey's Theorem and the existence of Infinite Chains or Infinite Anti-Chains in Infinite Posets, The Journal of Symbolic Logic. 81 (2016). no. 1, pp. 384-394, DOI: 10.1017/jsl.2015.47.

[Tac16b] _. On Martins Axiom and Forms of Choice, MLQ Math. Log. Q. 62 (2016), no. 3, pp. 190-203.

[THS16] E. Tachtsis, P. Howard, and D.I. Saveliev, On the set-theoretic strength of the existence of disjoint cofinal sets in posets without maximal elements, MLQ Math. Log. Q. 62 (2016), no. 3, pp. 155-176. DOI: 10.1002/malq.201400089.

Department of Logic, Institute of Philosophy, Eötvös Loránd University, Múzeum krt. 4/i Budapest, H-1088 HUNGARY

E-mail address: banerjee.amitayu@gmail.com

Institute of Philosophy, Department of Logic, Jagiellonian University, Grodzka 52, 33-332, Kraków, POLAND

E-mail address: zalan.gyenis@gmail.com 